\title{
STRUCTURE OF ESSENTIAL SPECTRA AND DISCRETE SPECTRUM OF THE ENERGY OPERATOR OF SIX ELECTRON SYSTEMS IN THE HUBBARD MODEL. FIRST QUINTET STATE \\ @ S.M. Tashpulatov
}

sadullatashpulatov@yandex.com,toshpul@mail.ru

\author{
УДК 517.984
}

DOI: 10.33184/mnkuomsh1t-2021-10-06.29.

Рассматривается шестиэлектронная система в модели Хаббарда. Описана структура существенного спектра и дискретный спектр в этой модели в случае первого квинтетного состояния. Доказывается, что, в одномерном случае, существенный спектр системы есть объединение семьи отрезков, а дискретный спектр системы состоит из не более одного собственного значения.

Ключевые слова: модель Хаббарда, шестиэлектронная система, квинтетное состояние, существенный спектр, дискретный спектр.

We consider of the energy operator of six electron systems in the Hubbard model and investigated the structure of essential spectra and discrete spectrum of the system in the first quintet state. We proved, that the if $\nu=1$, then the essential spectra of the operator is consists of the union of seven segments, and discrete spectrum of the operator is consists of no more then one eigenvalue.

Keywords: Hubbard model,six electron systems, quintet state, essential spectra, discrete spectrum.

The Hubbard model model is currently one of the most extensively studied multielectron models of metals $[1,2]$. But little is known about exact results for the spectrum and wave functions of the crystal described by the Hubbard model, and obtaining the corresponding statements is great interest problem.

Consider the energy operator $H$ of six-electron systems in the Hubbard model, where

$$
H=A \sum_{m, \gamma} a_{m, \gamma}^{+} a_{m, \gamma}+B \sum_{m, \tau, \gamma} a_{m, \gamma}^{+} a_{m+\tau, \gamma}+U \sum_{m} a_{m, \uparrow}^{+} a_{m, \uparrow} a_{m, \downarrow}^{+} a_{m, \downarrow} .
$$

Here $A$ is the electron energy at a lattice site, $B$ is the is the transfer integral between neighboring sites (we assume that $B>0$ for convenience),

Sa'dulla Tashpulatov (Doctor of Physics and Mathematics, Leading Researcher, Institute of Nuclear Physics of Academy of Sciences of Republic of Uzbekistan, Tashkent, Uzbekistan) 
$\tau= \pm e_{j}, j=1,2, \ldots, \nu$, where $e_{j}$ are unit mutually orthogonal vectors, which means that summation is taken over the nearest neighbors, $U$ is the parameter of the on-site Coulomb interaction of two electrons, $\gamma$ is the spin index, $\gamma=\uparrow$ or $\gamma=\downarrow, \uparrow$ and $\downarrow$ denote the spin values $\frac{1}{2}$ and $-\frac{1}{2}$, and $a_{m, \gamma}^{+}$ and $a_{m, \gamma}$ are the respective electron creation and annihilation operators at a site $m \in Z^{\nu}$.

The first quintet state corresponds six-electron bound states (or antibound states) to the basis functions: ${ }^{1} q_{p, n, r, t, l, k \in Z^{\nu}}^{2}=a_{p, \downarrow}^{+} a_{n, \uparrow}^{+} a_{r, \uparrow}^{+} a_{t, \uparrow}^{+} a_{l, \uparrow}^{+} a_{k, \uparrow}^{+} \varphi_{0}$. The subspace ${ }^{1} \widetilde{\mathcal{H}}_{q}^{2}$, corresponding to the first quintet state is the set of all vectors of the form ${ }^{1} \psi_{q}^{2}=\sum_{p, n, r, t, l, k \in Z^{\nu}} f(p, n, r, t, l, k)^{1} q_{p, n, r, t, l, k}^{2}, f \in l_{2}^{a s}$, where $l_{2}^{a s}$ is the subspace of antisymmetric functions in $l_{2}\left(\left(Z^{\nu}\right)^{6}\right)$. In this case, the Hamiltonian $H$ acts in the antisymmetric Foc'k space $\widetilde{\mathcal{H}}_{a s}$. Let $\varphi_{0}$ be the vacuum vector in the antisymmetrical Foc'k space $\widetilde{\mathcal{H}}_{a s}$. Let ${ }^{1} \widetilde{H}_{q}^{2}$ be the restriction $H$ to the subspace ${ }^{1} \widetilde{\mathcal{H}}_{q}^{2}$. The first quintet state corresponds the free motions of six-electrons in the lattice and their interactions.

Theorem 1. The subspace ${ }^{1} \mathcal{H}_{2}^{q}$ is invariant under the operator $H$, and the operator ${ }^{1} H_{2}^{q}$ is a bounded self-adjoint operator. It generates a bounded self-adjoint operator ${ }^{1} \bar{H}_{2}^{q}$ acting in the space $l_{2}^{a s}$ as

$$
\begin{gathered}
{ }^{1} \bar{H}_{2}^{q} \psi_{2}^{q}=6 A f(p, n, r, t, l, k)+B \sum_{\tau}[f(p+\tau, n, r, t, l, k)+f(p, n+\tau, r, t, l, k)+ \\
+f(p, n, r+\tau, t, l, k)+f(p, n, r, t+\tau, l, k)+f(p, n, r, t, l+\tau, k)+ \\
+f(p, n, r, t, l, k+\tau)]+U\left[\delta_{p, n}+\delta_{p, r}+\delta_{p, t}+\delta_{p, l}+\delta_{p, k}\right] f(p, n, r, t, l, k) .
\end{gathered}
$$

The operator ${ }^{1} H_{2}^{q}$ acts on a vector ${ }^{1} \psi_{2}^{q} \in{ }^{1} \mathcal{H}_{2}^{q}$ as

$$
{ }^{1} H_{2}^{q}{ }^{1} \psi_{2}^{q}=\sum_{p, n, r, t, l, k \in Z^{\nu}}\left({ }^{1} \bar{H}_{2}^{q} f\right)(p, n, r, t, l, k){ }^{1} \psi_{2}^{q} .
$$

We set ${ }^{1} \widetilde{H}_{2}^{q}=\mathcal{F}^{1} \bar{H}_{2}^{q} \mathcal{F}^{-1}$. In the quasimomentum representation, the operator ${ }^{1} \widetilde{H}_{2}^{q}$ acts in the Hilbert space $L_{2}^{a s}\left(\left(T^{\nu}\right)^{6}\right)$ as

$$
\begin{gathered}
{ }^{1} \widetilde{H}_{2}^{q 1} \psi_{2}^{q}= \\
\left\{6 A+2 B \sum_{i=1}^{\nu}\left[\cos \lambda_{i}+\cos \mu_{i}+\cos \gamma_{i}+\cos \theta_{i}+\cos \eta_{i}+\cos \xi_{i}\right]\right\} f(\lambda, \mu, \gamma, \theta, \eta, \xi)+ \\
+U \int_{T^{\nu}}[f(s, \lambda+\mu-s, \gamma, \theta, \eta, \xi)+f(s, \mu, \lambda+\gamma-s, \theta, \eta, \xi)+f(s, \mu, \gamma, \lambda+\theta-s, \eta, \xi)+ \\
+f(s, \mu, \gamma, \theta, \lambda+\eta-s, \xi)+f(s, \mu, \gamma, \theta, \eta, \lambda+\xi-s)] d s
\end{gathered}
$$


where $L_{2}^{a s}\left(\left(T^{\nu}\right)^{6}\right)$ is the subspace of antisymmetric functions in $L_{2}\left(\left(T^{\nu}\right)^{6}\right)$.

Theorem 2. Let $\nu=1$, and $U<0$. Then the essential spectrum of the operator ${ }^{1} \widetilde{H}_{2}^{q}$ is consists of the union of seven segments: $\sigma_{\text {ess }}\left({ }^{1} \widetilde{H}_{2}^{q}\right)=$ $[a+c+e, b+d+f] \cup\left[a+c+z_{3}, b+d+z_{3}\right] \cup\left[a+e+z_{2}, b+f+z_{2}\right] \cup\left[a+z_{2}+z_{3}, b+z_{2}+\right.$ $\left.z_{3}\right] \cup\left[c+e+z_{1}, d+f+z_{1}\right] \cup\left[c+z_{1}+z_{3}, d+z_{1}+z_{3}\right] \cup\left[e+z_{1}+z_{2}, f+z_{1}+z_{2}\right]$, and the discrete spectrum of operator ${ }^{1} \widetilde{H}_{2}^{q}$ is consists of no more one eigenvalue: $\sigma_{\text {disc }}\left({ }^{1} \widetilde{H}_{2}^{q}\right)=\left\{z_{1}+z_{2}+z_{3}\right\}$, or $\sigma_{\text {disc }}\left({ }^{1} \widetilde{H}_{2}^{q}\right)=\emptyset$, Here and hereafter $a=$ $2 A-4 B \cos \frac{\Lambda_{1}}{2}, b=2 A+4 B \cos \frac{\Lambda_{1}}{2}, c=2 A-4 B \cos \frac{\Lambda_{2}}{2}, d=2 A+4 B \cos \frac{\Lambda_{2}}{2}$, $e=2 A-4 B \cos \frac{\Lambda_{3}}{2}, f=2 A+4 B \cos \frac{\Lambda_{3}}{2}, z_{1}=2 A-2 \sqrt{U^{2}+4 B^{2} \cos ^{2} \frac{\Lambda_{1}}{2}}$, $z_{2}=2 A+2 \sqrt{U^{2}+4 B^{2} \cos ^{2} \frac{\Lambda_{2}}{2}}$, and $z_{3}=2 A-\sqrt{U^{2}+16 B^{2} \cos ^{2} \frac{\Lambda_{3}}{2}}$. Here $\Lambda_{1}=\lambda+\mu, \Lambda_{2}=\gamma+\theta, \Lambda_{3}=\eta+\xi$

Theorem 3. Let $\nu=1$, and $U>0$. Then the essential spectrum of the operator ${ }^{1} \widetilde{H}_{2}^{q}$ is consists of the union of seven segments: $\sigma_{\text {ess }}\left({ }^{1} \widetilde{H}_{2}^{q}\right)=$ $[a+c+e, b+d+f] \cup\left[a+c+\widetilde{z}_{3}, b+d+\widetilde{z}_{3}\right] \cup\left[a+e+\widetilde{z}_{2}, b+f+\widetilde{z}_{2}\right] \cup[a+$ $\left.\widetilde{z}_{2}+\widetilde{z}_{3}, b+\widetilde{z}_{2}+\widetilde{z}_{3}\right] \cup\left[c+e+\widetilde{z}_{1}, d+f+\widetilde{z}_{1}\right] \cup\left[c+\widetilde{z}_{1}+\widetilde{z}_{3}, d+\widetilde{z}_{1}+\widetilde{z}_{3}\right] \cup[e+$ $\left.\widetilde{z}_{1}+\widetilde{z}_{2}, f+\widetilde{z}_{1}+\widetilde{z}_{2}\right]$, and the discrete spectrum of operator ${ }^{1} \widetilde{H}_{2}^{q}$ is consists of no more one eigenvalue: $\sigma_{\text {disc }}\left({ }^{1} \widetilde{H}_{2}^{q}\right)=\left\{\widetilde{z}_{1}+\widetilde{z}_{2}+\widetilde{z}_{3}\right\}$, or $\sigma_{\text {disc }}\left({ }^{1} \widetilde{H}_{2}^{q}\right)=\emptyset$. Here $\widetilde{z}_{1}=2 A+2 \sqrt{U^{2}+4 B^{2} \cos ^{2} \frac{\Lambda_{1}}{2}}, \widetilde{z}_{2}=2 A-2 \sqrt{U^{2}+4 B^{2} \cos ^{2} \frac{\Lambda_{2}}{2}}$, and $\widetilde{z}_{3}=2 A+\sqrt{U^{2}+16 B^{2} \cos ^{2} \frac{\Lambda_{3}}{2}}$.

\section{Литература}

1. Hubbard J. Electron Correlations in Narrow Energy Band // Proc. Roy. Soc. A. 276:1365 (1963), 238- 257.

2. Karpenko B.V., Dyakin V.V., and Budrina G.L. Two electrons in the Hubbard Model.// Phys. Met. Metallogr. 61 (1986), 702-706. 